Phys. kondens. Materie 14, 37-54 (1971)

(C) by Springer-Verlag 1971

\title{
Crystallographic, Optical and Magnetic Properties of $\mathrm{Eu}_{2} \mathrm{SiO}_{4}$
}

\author{
R. VERREAULT * \\ Laboratorium für Festkörperphysik ETH \\ Zurich, Switzerland
}

Received January 1, 1971

\begin{abstract}
The crystallographic properties of $\mathrm{Eu}_{2} \mathrm{SiO}_{4}$ are studied in terms of its isomorph $\mathrm{Ca}_{2} \mathrm{SiO}_{4}$. The recently discovered monoclinic room-temperature phase is ferroelastic and simultaneously ferromagnetic at low temperatures $\left(T_{\mathbf{c}}=5.40^{\circ} \mathrm{K}\right)$. The optical absorption and the dispersion properties have been measured in spectral intervals ranging from 0.5 to $3.6 \mathrm{eV}$ and partly for temperatures between 300 and $500^{\circ} \mathrm{K}$. This temperature range includes the ferroelasticparaelastic phase-transition temperature $\left(T_{\mathrm{e}}=438^{\circ} \mathrm{K}\right)$. An anomaly of the dielectric constant at $T_{\mathrm{e}}$ suggests the presence of an unstable phase which would be ferroelectric. The Faraday rotation has been measured on either side of the absorption edge at 300 and $77^{\circ} \mathrm{K}$. The recent results on crystal structure allow an explanation of the magnetic behaviour of the two ferromagnetic phases known up to now.
\end{abstract}

Die kristallographischen Eigenschaften von $\mathrm{Eu}_{2} \mathrm{SiO}_{4}$ werden in Zusammenhang mit dem isomorphen $\mathrm{Ca}_{2} \mathrm{SiO}_{4}$ untersucht. Die neulich gefundene monokline Zimmertemperaturphase ist ferroelastisch und zugleich ferromagnetisch bei tiefen Temperaturen $\left(T_{\mathbf{c}}=5,40^{\circ} \mathrm{K}\right)$. Die optische Absorption und die Dispersionseigensehaften wurden in Spektralbereichen zwischen. 0,5 und $3,6 \mathrm{eV}$ und zum Teil für Temperaturen zwischen 300 und $500^{\circ} \mathrm{K}$ gemessen. Dieser Temperaturbereich schließt die ferroelastisch-paraelastische Phasenumwandlungstemperatur ein $\left(T_{\mathrm{e}}=438^{\circ} \mathrm{K}\right)$. Eine Anomalie der DK deutet auf die Existenz einer unstabilen ferroelektrischen Phase hin. Die Faraday-Drehung wurde auf beiden Seiten der Absorptionskante bei 300 und $77^{\circ} \mathrm{K}$ gemessen. Die neuesten Angaben über die Kristallstruktur ermöglichen eine Erklärung des magnetischen Verhaltens der zwei bis jetzt bekannten ferromagnetischen Phasen.

Nous présentons une étude des propriétés cristallographiques de $\mathrm{Eu}_{2} \mathrm{SiO}_{4}$ en regard de son isomorphe $\mathrm{Ca}_{2} \mathrm{SiO}_{4}$. La phase monoclinique découverte récemment comme étant stable à la température ambiante est ferro-élastique et de plus ferromagnétique aux basses températures $\left(T_{\mathrm{c}}=5,40^{\circ} \mathrm{K}\right)$. Nous avons mesuré l'absorption optique et les propriétés de dispersion dans des intervalles spectraux s'étendant de 0,5 à $3,6 \mathrm{eV}$ et en partie à des températures s'échelonnant entre 300 et $500^{\circ} \mathrm{K}$. Ce domaine thermique comprend la température de transition entre les phases ferro-élastique et para-élastique $\left(T_{\mathrm{e}}=438^{\circ} \mathrm{K}\right)$. Une anomalie de la constante diélectrique à $T_{\mathrm{e}}$ suggère la présence d'une phase instable ferro-électrique. Nous avons mesuré la rotation de Faraday de part et d'autre de l'arête d'absorption à 300 et $77^{\circ} \mathrm{K}$. Les résultats obtenus récemment sur la structure cristalline fournissent une explication du comportement magnétique des deux phases ferromagnétiques présentement connues.

\section{Introduction}

The discovery of ferromagnetism in insulating $\mathrm{CrBr}_{3}$ [1] in 1960 and especially the discovery of ferromagnetic EuO one year later [2] marked the start of an intensive search for magnetically ordering semi-conductors. A systematic investigation of the cubic rare-earth compounds showed in 1962 that magnetic ordering existed in the other chalcogenides of divalent europium [3]. The chalcogenide series

* Now at Université du Québec, Chicoutimi, Qué., Canada. 
was especially attractive, since it allowed a study of the Heisenberg ferromagnet with a minimum of disturbing factors, namely in the simple sodium chloride structure and with the magnetic $4 f$-electrons not being involved in the binding mechanisms of the substance. Due to their semi-conducting behaviour, the Eu-chalcogenides lend themselves naturally to the study of magnetism by optical means. In particular the interesting phenomenon of the magnetic red shift of the absorption edge [4] was found to give a direct measure of the magnetic splitting of the conduction band due to the spontaneous or induced ferromagnetic ordering at low temperatures.

Among the semi-conducting compounds of divalent europium in which magnetic ordering was found during the last decade, europium orthosilicate $[5,6]$ did not captivate the general interest. Apart from the difficulty of obtaining it in a pure single-crystal form, some of the reasons appear to be the rather complicated expected structure and the optical anisotropy, which is annoying in magnetooptical studies [7]. However, the recent production of fairly pure single crystals [8] and the discovery of a new phase, stable below $165^{\circ} \mathrm{C}[9]$, have allowed a good deal of optical and crystallographic data to be collected.

By comparing the ordering temperature of $\mathrm{Eu}_{2} \mathrm{Si}_{4}$ with those of the chalcogenides and assuming a 6-fold coordination, Shafer et al. [7] have proposed that the Eu-Eu distances in the silicate should be of the same magnitude as those of EuSe, namely 4.4 and $6.2 \AA$ for the nearest and the next-nearest neighbours respectively. It will be seen below however that the dissimilarities in crystal structure lead to a drastically different situation. In this paper, we present at first some new data concerning the crystallographic structure of both the orthorhombic and the monoclinic phases. Then a study of the optical properties with emphasis on characterization is reported and finally an estimate of the magnetic exchange parameters is made in terms of the available information on the structure and of the molecular field approximation.

\section{Crystallographic properties}

A room-temperature phase of $\mathrm{Eu}_{2} \mathrm{SiO}_{4}$ has been indexed by $\mathrm{Rau}[10]$ as orthorhombic with a superstructure along the $b$ axis and hence the lattice constants $a=9.713, \mathrm{~b}=49.56$ and $c=\mathbf{5 . 6 5 2} \AA$. In contrast with these results, the roomtemperature and high-temperature phases of the single crystals grown in this laboratory have been indexed by Busch et al. [9] as belonging to the respective space groups $\mathrm{P} 2_{1} / \mathrm{c}$ (monoclinic) and Pmon (orthorhombic). A more convenient space group setting in each phase, namely $\mathrm{P}_{1} / \mathrm{n}$ and Pmnb, may be justified by the properties reported below and the unit cell dimensions corresponding to these space groups are given in Table 1 . It may be noted that none of the superstructure reflexes reported by Rau has been observed in our substance.

\subsection{Structure}

The space groups found for $\mathrm{Eu}_{2} \mathrm{SiO}_{4}$ are those of monoclinic $\beta$-Can $\mathrm{CiO}_{4}$ and orthorhombic $\alpha^{\prime}-\mathrm{Ca}_{2} \mathrm{SiO}_{4}$, the respective structures of which have been determined by Midgley [11] and Yamaguchi et al. [12]. The structure determination of monoclinic $\mathrm{Eu}_{2} \mathrm{SiO}_{4}$ by Felsche [13] has confirmed the isomorphism with $\beta$ - $\mathrm{Ca}_{2} \mathrm{SiO}_{4}$ in 
Table 1. Comparative crystallographic data of $\mathrm{Eu}_{2} \mathrm{SiO}_{4}$ and $\mathrm{Ca}_{2} \mathrm{SiO}_{4}$

\begin{tabular}{|c|c|c|c|c|}
\hline & \multicolumn{2}{|l|}{ monoclinic $\left(\mathrm{P} 2_{1} / \mathrm{n}\right)$} & \multicolumn{2}{|c|}{ orthorhombic (Pmnb) } \\
\hline & $\mathrm{Eu}_{2} \mathrm{SiO}_{4}$ & $\beta-\mathrm{Ca}_{2} \mathrm{SiO}_{4}$ & $\mathrm{Eu}_{2} \mathrm{SiO}_{4}$ & $\alpha^{\prime}-\mathrm{Ca}_{2} \mathrm{SiO}_{4}$ \\
\hline$a[\AA]$ & 5.6513 & 5.48 & 5.665 & 5.491 \\
\hline$b$ & 7.0949 & 6.76 & 7.137 & 6.790 \\
\hline$c$ & 9.7637 & 9.28 & 9.767 & 9.248 \\
\hline$\beta$ & $92.57^{\circ}$ & $94.55^{\circ}$ & & \\
\hline$a b c \sin \beta\left[\AA^{3}\right]$ & 391.1 & 342.7 & 394.8 & 344.8 \\
\hline$a: b: c$ & $0.797: 1: 1.487$ & $0.810: 1: 1.491$ & $0.795: 1: 1.495$ & $0.808: 1: 1.502$ \\
\hline $\begin{array}{l}\text { cation radius } \\
\text { (Pauling) }\lceil\AA]\end{array}$ & 1.12 & 0.99 & & \\
\hline reference & $\begin{array}{l}\text { Felsche } \\
(1970)\end{array}$ & $\begin{array}{l}\text { Midgley } \\
(1952)\end{array}$ & $\begin{array}{l}\text { Felsche } \\
(1970)\end{array}$ & $\begin{array}{l}\text { Yamaguchi } \\
(\mathbf{1 9 5 7 )}\end{array}$ \\
\hline
\end{tabular}

so far as the cation positions are concerned. However, the oxygen positions in both substances are somewhat unreliable due to imperfections [11] or unfavourable geometry [13] of the investigated crystals. The isomorphism between $\mathrm{Eu}_{2} \mathrm{SiO}_{4}$ and $\mathrm{Ca}_{2} \mathrm{SiO}_{4}$ for the two phases considered appears nevertheless very plausible, as shown by the comparison of their properties. This implies that the orthorhombic structure of $\mathrm{Eu}_{2} \mathrm{SiO}_{4}$ would be of the $\beta$ - $\mathrm{K}_{2} \mathrm{SO}_{4}$ type. Without attempting to impose any nomenclature or to correct an apparently unfortunate one for $\mathrm{Ca}_{2} \mathrm{SiO}_{4}$, the phase designations of the latter will be used in this work for the corresponding polymorphs of $\mathrm{Eu}_{2} \mathrm{SiO}_{4}$.

In the orthorhombic phase, all the $\mathrm{Eu}^{2+}$ and $\mathrm{Si}^{4+}$ ions lie in the planes $x= \pm 1 / 4$ and the $\mathrm{SiO}_{4}$ tetrahedra have their base flat on a (010) plane and one edge along the $a$ axis. The monoclinic cell is then derived from the orthorhombic one by a collective rotation of the tetrahedra and by minute displacements $(0.05-0.11 \AA)$ of the $\mathrm{Eu}^{2+}$ and $\mathrm{Si}^{4+}$ ions out of those planes.

Both the $\alpha^{\prime}$ and $\beta$ phases can be visualized as consisting of chains of europiumtetrahedron units aligned along the $b$ axis. The $\mathrm{Eu}^{2+}$ ions belonging to the chains (hereafter called $\mathrm{E} u_{I}$ ) sit between the apex of a tetrahedron and the base of the following one. The various chains are juxtaposed along the $\alpha$ axis in alternating upward and downward orientations and the sheets thus formed are stacked behind one another along the $c$ axis in a manner which respects the symmetry elements of the pertaining space group, i.e. turned each time about the screw dyad axis $(x=1 / 4, z=1 / 4)$ and shifted by $b / 2$ in the $y$ direction. The free spaces between the sheets can then all be filled by an equal number of additional $\mathbf{E u}{ }^{2+}$ ions $\left(\mathbf{E u}_{\mathrm{II}}\right)$. The positions of $\mathbf{E u}_{\mathrm{I}}$ and $\mathrm{Eu}_{\mathrm{II}}$ are crystallographically unequivalent. It may be pointed out that the above concept of sheet does not correspond to the well known layer structures encountered in many silicates since one is dealing here with discrete tetrahedra. It appears however to be justified by the easy cleavage of $\beta$-Eu $\mathrm{ESiO}_{4}$ along (001) planes.

Comparative crystallographic data of $\mathrm{Ca}_{2} \mathrm{SiO}_{4}$ and $\mathrm{Eu}_{2} \mathrm{SiO}_{4}$ with the mutually related space groups $\mathbf{P} 2_{1} / \mathbf{n}$ and Pmnb have been collected in Table 1 . In both substances, there is a similar very small volume change at the phase transition $\left(165^{\circ} \mathrm{C}\right.$ for $\mathrm{Eu}_{2} \mathrm{SiO}_{4}$ and $670^{\circ} \mathrm{C}$ for $\left.\mathrm{Ca}_{2} \mathrm{SiO}_{4}\right)$. Among the properties obviously arising from the different cation radii, one has the differences in the angle $\beta$ and 


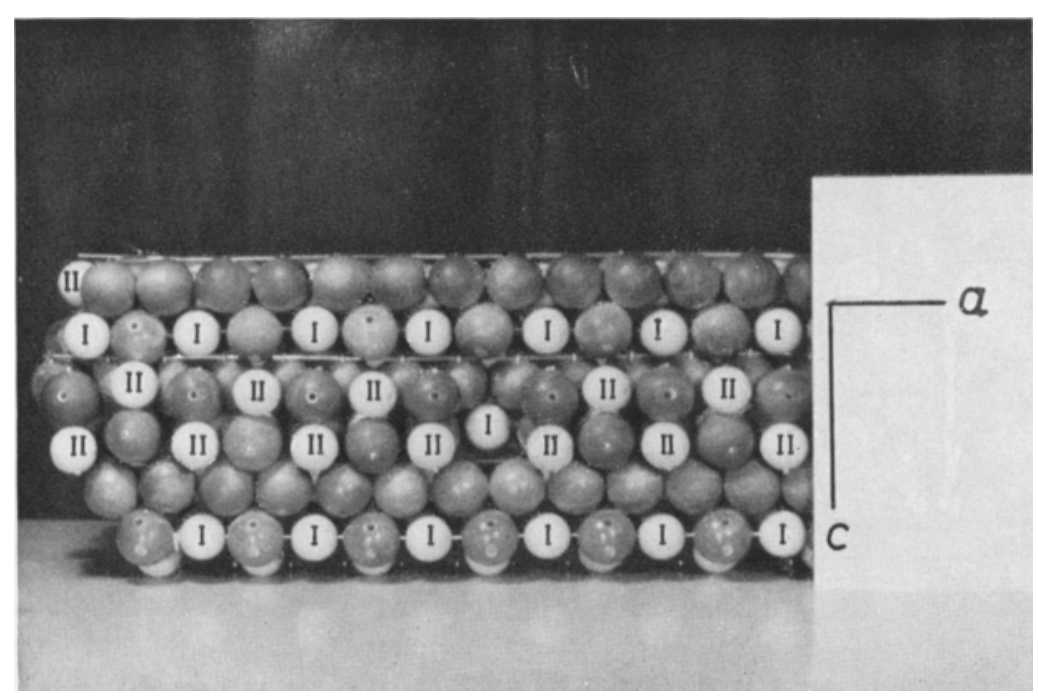

Fig. 1. Elastically bonded model of $\mathrm{Eu}_{2} \mathrm{SiO}_{4}$ viewed along the $b$ axis in the orthorhombic modification. Bright spheres: $\mathrm{Eu}^{2+}$; dark spheres: $\mathrm{O}^{2-} \cdot\left(\mathrm{Si}^{4+}\right.$ is not shown)

particularly in the axial ratios $c: b$ and $a: b$. The latter is $1.5 \%$ smaller for $\mathrm{Eu}_{2} \mathrm{SiO}_{4}$ in both phases, whereas the average linear dilatation is $4.5 \%$. This suggests that the structure just described has much available space for cations of various sizes in a $(010)$ plane, which is also consistent with the statement by Midgley [11] that the cell of $\beta$ - $\mathrm{Ca}_{2} \mathrm{SiO}_{4}$ is compressed along the $b$ axis. The amount of rotation of the tetrahedra and consequently the departure of the angle $\beta$ from $90^{\circ}$ would then be determined by the contact conditions between the cations and a certain number of their neighbouring $\mathrm{O}^{2-}$ ions. They should therefore be both larger for smaller cations.

The same reasoning can explain the experimental fact [14] that in spite of the $\alpha^{\prime}-\beta$ phase transition of $\mathrm{Ca}_{2} \mathrm{SiO}_{4}$ at $670^{\circ} \mathrm{C}$ a small percentage of $\mathrm{B}_{2} \mathrm{O}_{3}$ in solid solution suffices to stabilize the $\alpha^{\prime}$ phase at room temperature without affecting the X-ray data. It can be argued that the large additional $\mathrm{O}^{2-}$ ions sit on former $\mathrm{Ca}^{2+}$ sites between tetrahedra in the vicinity of the much smaller $\mathrm{B}^{3+}$ ions and thus hinder the collective rotating of the tetrahedra in a sufficiently large volume to prevent the bulk from changing phase. It is therefore probable that the roomtemperature orthorhombic phase of $\mathrm{Eu}_{2} \mathrm{SiO}_{4}$ studied by Shafer et al. [7] was stabilized by the presence of some at. \% of $\mathrm{Eu}^{3+}$ in the form of dissolved $\mathrm{Eu}_{2} \mathrm{O}_{3}$ and that the coupling of such distorted centers in higher concentration give rise to microtwinning and superstructure reflexes as observed by Rau.

The consistency of the foregoing speculations has been partly verified on a close-packed scale model of $\mathrm{Eu}_{2} \mathrm{SiO}_{4}$ based on Pauling's ionic radii. It was built out of rigid-tetrahedron units and loose spheres elastically bonded to the tetrahedra. This model was therefore capable of simulating the $\alpha^{\prime}-\beta$ phase transition. Fig. 1 shows the orthorhombic modification in a view along the $b$ axis. One sees for instance at the pseudo-hexagonal surroundings of $E u_{I}$ in the (010) plane that 


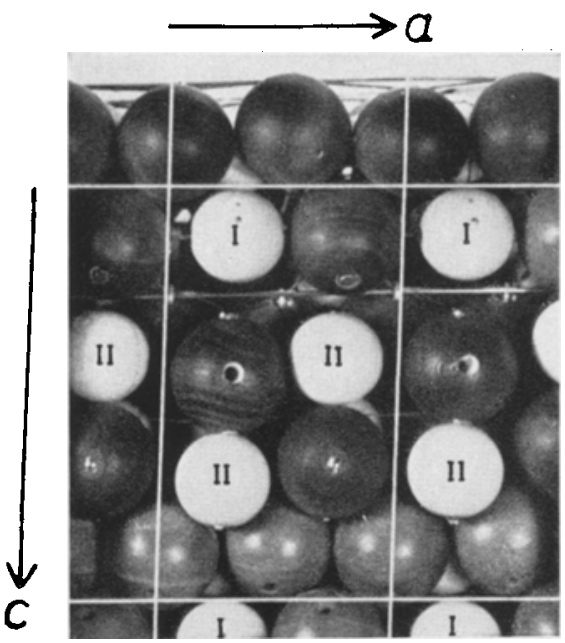

Fig. 2. Close up view of the same model in the monoclinic modification

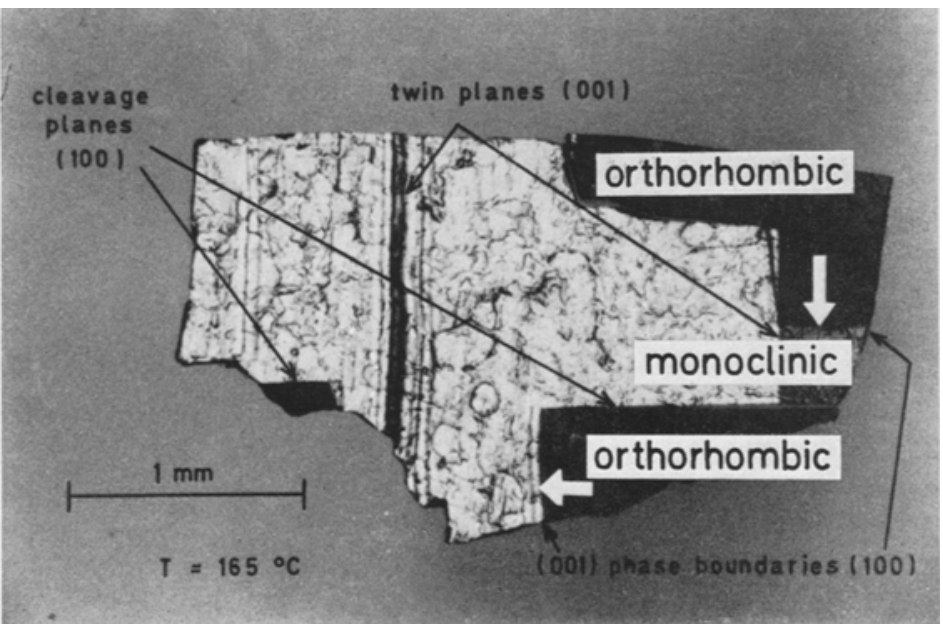

Fig. 3. Spontaneous cleavage of an as-grown platelet of $\mathrm{Eu}_{2} \mathrm{SiO}_{4}$ related to the ferroelasticparaelastic phase transition at $165^{\circ} \mathrm{C}$. The white arrows indicate the positions and the directions of motion of the phase boundaries. $(25 \times)$. The plane designation should be interchanged. Read (100) instead of (001), and vice versa

an ion as large as $\mathrm{O}^{2-}$ could be accomodated on that site. In the monoclinic modification (Fig. 2), the $\mathrm{Eu}^{2+}$ ions are circumscribed by the rotated tetrahedra in such a way that the corresponding equilibrium value for the angle $\beta$ equals $92^{\circ}$ on the model. This is in fair agreement with the $\mathrm{X}$-ray value.

\subsection{Ferroelasticity}

As reported earlier [9], the monoclinic phase of $\mathrm{Eu}_{2} \mathrm{SiO}_{4}$ shows polysynthetic twinning on (100) and (001). The mechanical and energetic behaviours of that twinning [15] make $\mathrm{Eu}_{2} \mathrm{SiO}_{4}$ belong to that special class of substances called by 


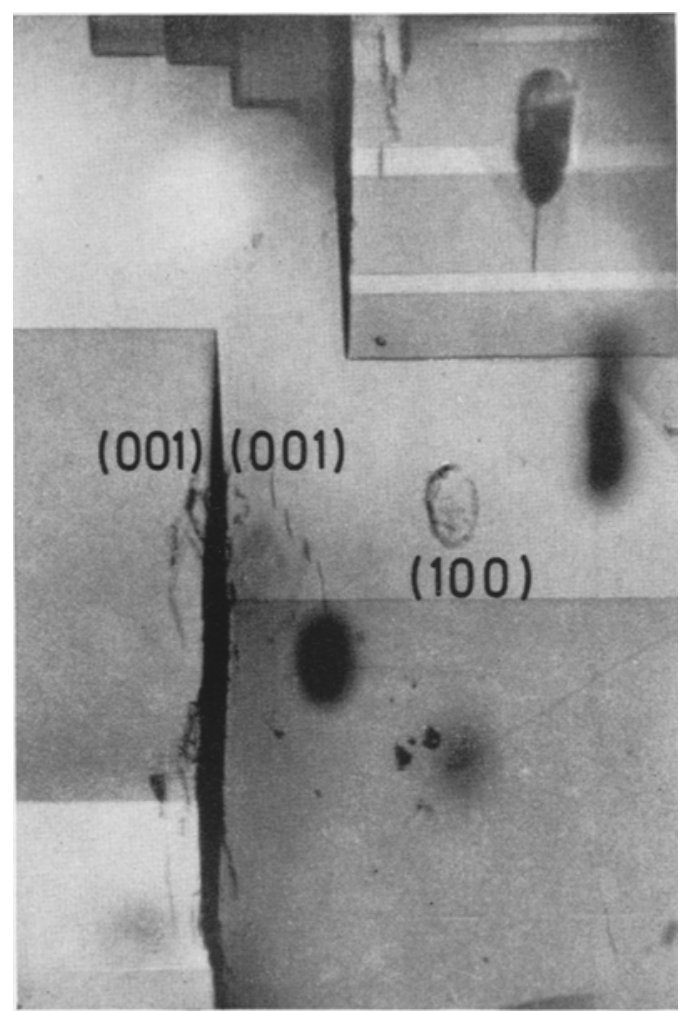

Fig. 4. Ferroelastic domains of $\beta$ - $\mathrm{Eu}_{2} \mathrm{SiO}_{4}$ viewed along the $b$ axis. The domain walls (twun planes) are along $(100)$ and the cleavage is on $(001)$. The opening angle $2 \beta-90^{\circ}=5.18^{\circ}$.

$$
(\sim 500 \times)
$$

Aizu [16] "ferroelastic crystals", namely in the ferroelastic non-ferroelectric species $\mathrm{mmmF} 2 / \mathrm{m}$. For each kind of twinning - on (100) or (001) - there exist only two states of different crystallographic orientation; they are enantiomorphs with respect to the twin plane and are derived from the common (prototypic) orthorhombic state by opposite components of atomic displacements in a direction normal to the twin plane. From energetical considerations, it comes out that the $\alpha^{\prime}-\beta$ phase transition is merely the paraelastic-ferroelastic transition, with an enthalpychange of $61 \mathrm{cal} / \mathrm{mole}$ [9]. This has been visually verified under the polarizing microscope (Fig. 3). On heating a twinned crystal through the transition temperature at a low rate $(\sim 0.1 \mathrm{deg} / \mathrm{min})$, the ferro-para phase boundary can be moved in a controllable way throughout the sample. The twin planes are then seen to disappear only when they are reached by the phase boundary, and on cooling, the new twin planes appear (in general at new positions) as if they were printed lines left behind by the moving phase boundary. Fig. 3 also illustrates how the $2.6^{\circ}$ angular difference between the $(001)$ planes of both phases shows up through a cleavage along those planes as the $(100)$ boundary proceeds normally to the common (100) plane. On the other hand, the (001) boundary, on the right, provoques an irregular rupture. The easy cleavage along (001) is also shown in Fig. 4, 

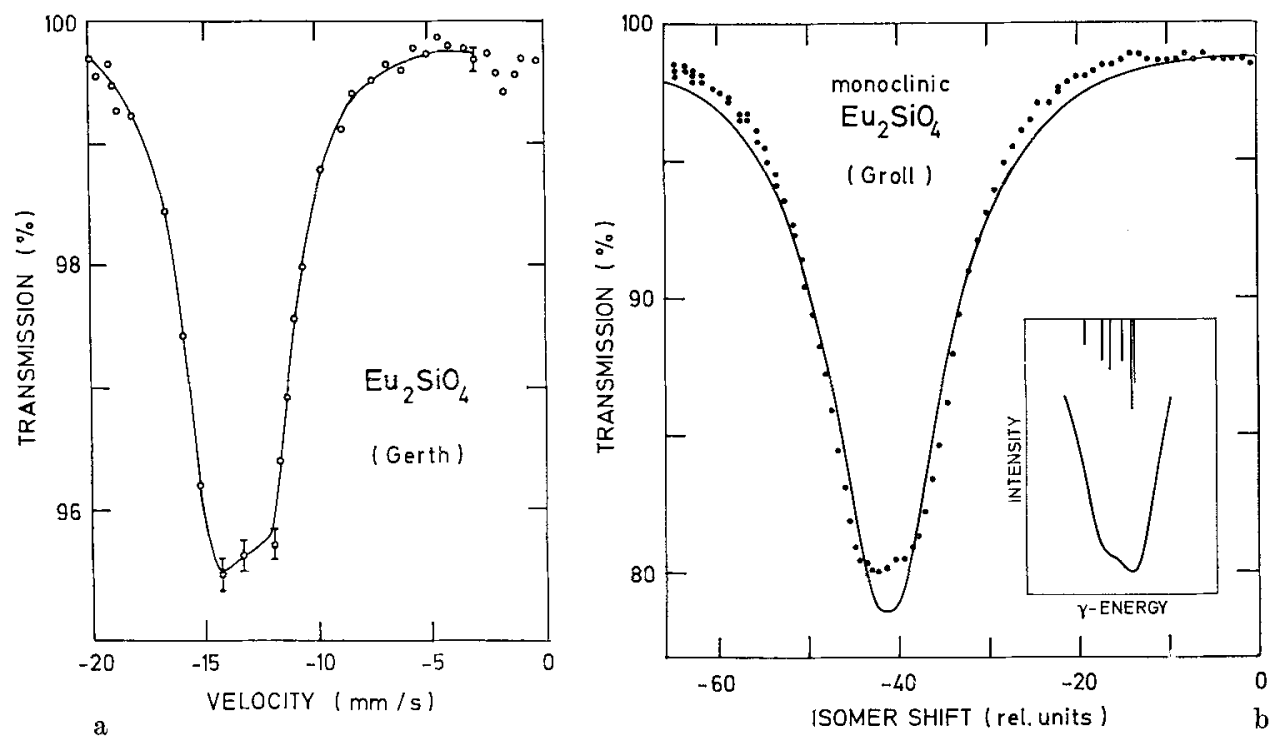

Fig. 5 a and b. Isomer shift in $\mathrm{Eu}_{2} \mathrm{SiO}_{4}$ : a) early measurements by Gerth [18], presumably on the $\alpha^{\prime}$ phase; b) measurements by Groll [20] on the $\beta$ phase. The dots are the experimental points and the solid curve shows the corresponding least-squares fit to a single line. The inset shows the line shape which would result from quadrupole splitting alone

which is a photomicrograph taken along the $b$ axis of the $\beta$ phase at room temperature. Here the various ferroelastic domains have been produced mechanically.

\subsection{Coordination}

The coordination of the cations in both the $\alpha^{\prime}$ and $\beta$ phases is irregular. The two unequivalent $\mathrm{Eu}^{2+}$ sites are charaeterized by different numbers of unshielded $\mathrm{O}^{2-}$ neighbours. On account of the fact that $\mathrm{Eu}_{2} \mathrm{SiO}_{4}$ has a definitely higher ionicity $(45 \%)$ ) than the chalcogenides [17], an estimate of the coordination from computed Eu-O distances and with the assumption of a purely electrostatic interaction gives approx. 8.5 for both $\mathrm{Eu}_{\mathrm{I}}$ and $\mathrm{Eu}_{\mathrm{II}}$ in the $\alpha^{\prime}$ phase, whereas the corresponding values of 8.4 and 9.0 have been obtained for the $\beta$ phase (Table 2 ). In agreement with this, Fig. 5 shows the isomer shift of $\mathrm{Eu}^{151}$ in $\mathrm{Eu}_{2} \mathrm{SiO}_{4}$. Gerth's measurements (Fig. 5a) [18] (1968) on a powder prepared by Pink [19] according

Table 2. Coordination data of $\mathrm{Eu}^{2+}$ ions in $\mathrm{Eu}_{2} \mathrm{SiO}_{4}$ $\bar{d}_{n n}$ is the mean distance of the $Z_{n n}$ nearest $\mathrm{O}^{2-}$ neighbours; $Z$ is the effective coordination number

\begin{tabular}{lllll}
\hline & \multicolumn{2}{l}{ monoclinic } & \multicolumn{2}{c}{ orthorhombic } \\
& Eu & EurI & Eu & Eu $_{\mathrm{II}}$ \\
\hline$Z_{n n}$ & 6 & 7 & 4 & 7 \\
$Z_{n n n}$ & $4-6$ & 2 & 6 & 2 \\
$\bar{d}_{n n}[\AA]$ & 2.5 & 2.5 & 2.5 & 2.5 \\
$\bar{d}_{n n n}[\AA]$ & 3.25 & 3.0 & 2.9 & 2.9 \\
$Z$ & 9 & 8.4 & 8.5 & 8.5 \\
\hline
\end{tabular}


to Shafer's method, and which is believed to be orthorhombic, indicated some structure of the divalent absorption peak but the statistics of the experiment were too unreliable to allow a definitive conclusion to be drawn. The trivalent peak however, near the origin, was neatly stronger than on the curve shown here for our $\beta$ phase, which was recently measured by Groll [20] (Fig. 5b). In the latter case, the $\mathrm{Eu}^{2+}$ absorption was significantly resolved in a peak with two shoulders. Since the line shape due to quadrupole splitting of the $27 \mathrm{keV}$ line used would show a steep rise one side of the minimum, the shoulder observed there can be ascribed to the presence of two unequivalent $\mathrm{Eu}^{2+}$ sites with different charge densities, and hence different coordinations, in the $\beta$ phase of $\mathrm{Eu}_{2} \mathrm{SiO}_{4}$.

\section{Optical Properties}

In order to characterize the room-temperature phase of $\mathrm{Eu}_{2} \mathrm{SiO}_{4}$, the optical absorption has been measured in polarized and unpolarized light over the energy range $0.5-3.6 \mathrm{eV}$, that is above and below the absorption edge at $2.5 \mathrm{eV}$. In contrast with the orthorhombic phase, the monoclinic one was found to be very strongly luminescent at room temperature. A study of the luminescent properties was given elsewhere $[9,21]$. A spontaneous shift of the absorption edge at the phase transition temperature has also been reported by Busch et al. [9]. In addition to the absorption results and to some magneto-optical measurements at room temperature and $77^{\circ} \mathrm{K}$, the present investigation includes a petrographic study of the $\beta$ phase pertaining to the dispersion of the indicatrix at room temperature. A peculiar behaviour in the temperature dependence of the static dielectric constant is also discussed.

\subsection{Optical Absorption}

\subsubsection{Transparent Region}

Fig. $6 \mathrm{a}$ shows some general absorption features of $\beta$ - $\mathrm{Eu}_{2} \mathrm{SiO}_{4}$ for unpolarized light at photon energies smaller than the energy gap. This part of the absorption

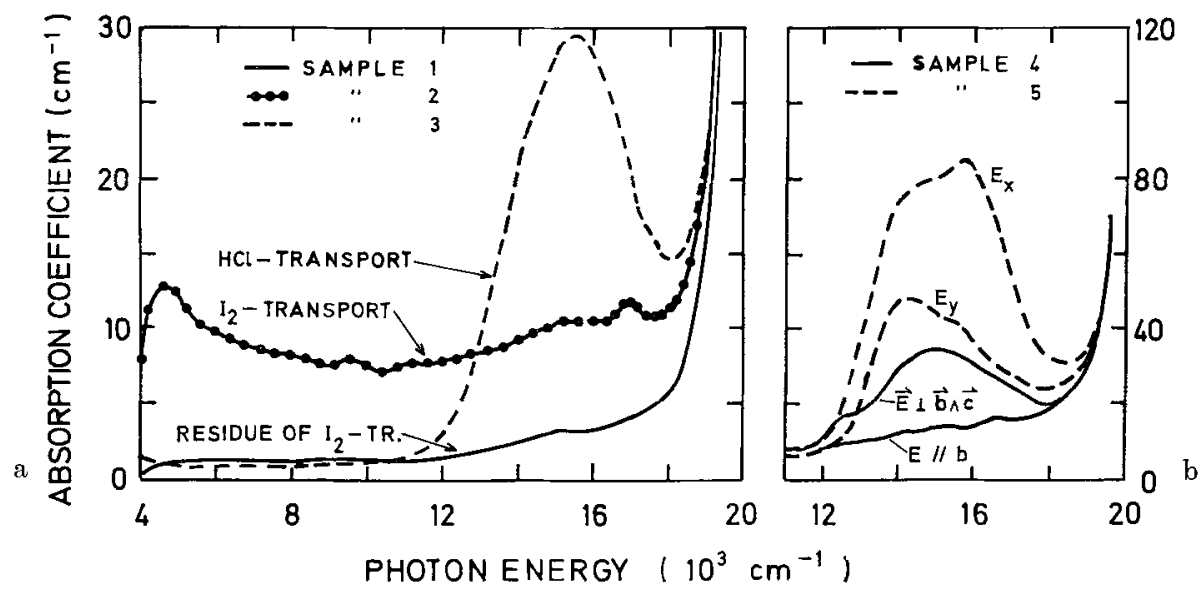

Fig. 6 a and b. Absorption spectrum of $\beta-\mathrm{Eu}_{2} \mathrm{SiO}_{4}$ in the transparent spectral region at room temperature: a) with unpolarized light; b) with linearly polarized light vibrations along the fast

$(\mathrm{Ox}, \boldsymbol{b} \wedge \boldsymbol{c})$ and slow $(\mathrm{Oy}, \boldsymbol{b})$ axes of two platelets 


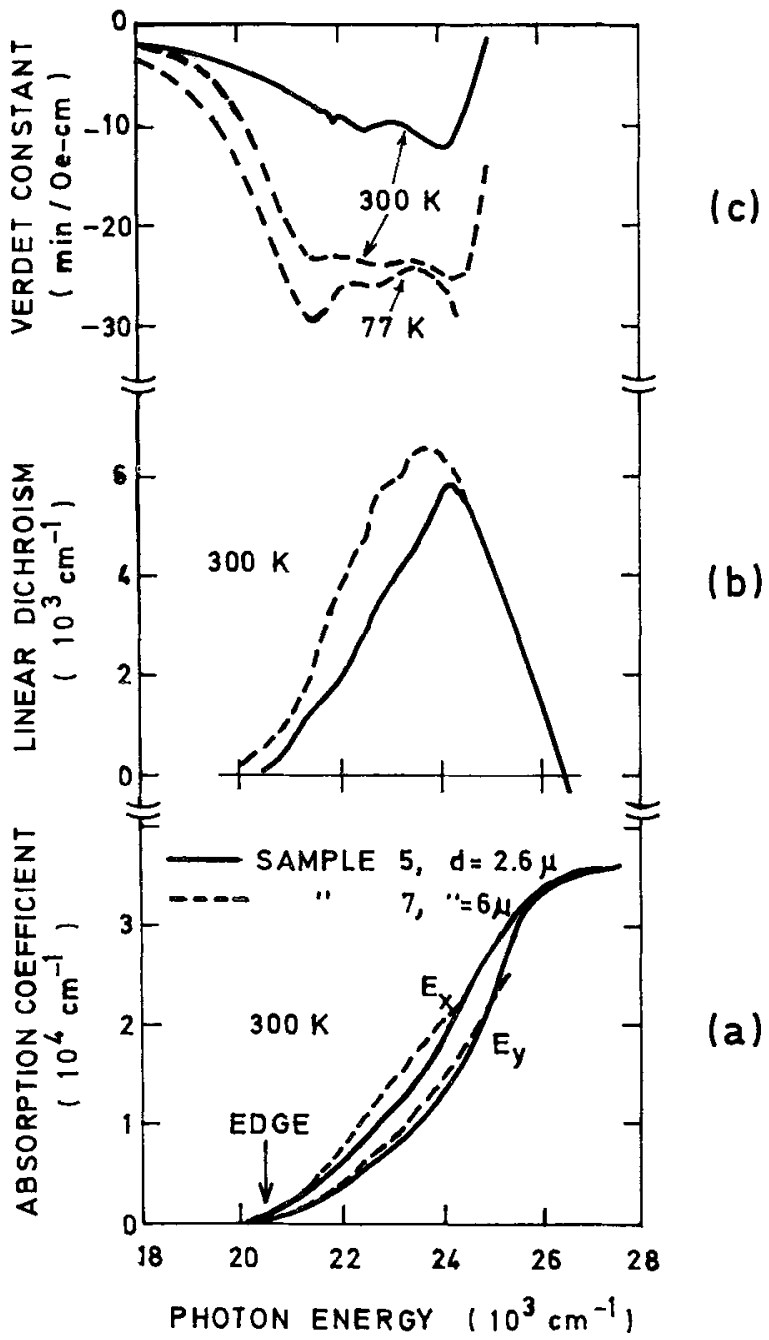

Fig. 7. Absorption coefficient, linear dichroism and Faraday rotation of $\beta$-Eu $\mathrm{SiO}_{4}$ in the vicinity of the absorption edge with linearly polarized light vibrations along the fast $(\mathrm{Ox})$ and slow (Oy) axes of each sample. Those samples were grown in thin platelet form; it was not possible to lap the crystals so as to obtain any desired face orientation

spectrum is mainly determined by impurity absorption. The simultaneous appearance of three peaks at 4800,17200 and $19000 \mathrm{~cm}^{-1}$ (sample 2) indicates the presence of $\mathrm{Eu}^{3+}$ ions in the lattice [22]. The third peak, which is mostly hidden in the absorption edge at room temperature, could be neatly resolved at low temperatures due to the corresponding blue-shift of the edge [23]. The type of impurity responsible for the absorption band of sample 3 in the red $\left(\sim 15000 \mathrm{~cm}^{-1}\right)$ has not yet been definitively clarified. As shown in Fig. 6 with polarized light, this band reveals a strong dichroism. It vanishes for light propagating along the $c$ axis and vibrating along the $b$ axis (sample 4 ), thus reflecting the monoclinic 


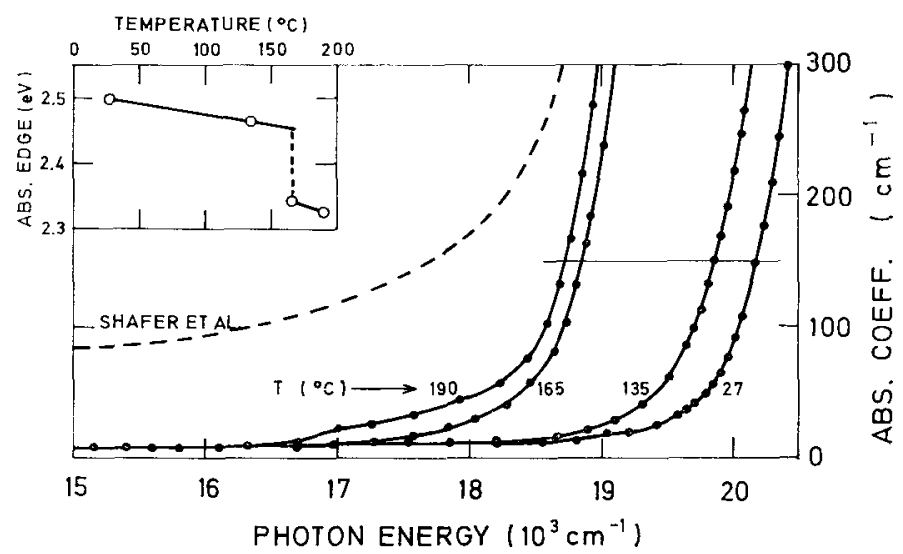

Fig. 8. Temperature dependence of the absorption edge of $\mathrm{Eu}_{2} \mathrm{SiO}_{4}$ about the $\alpha^{\prime}-\beta$ phase transition at $165^{\circ} \mathrm{C}$

crystal symmetry with its unique symmetry axis. For a general direction of propagation (sample 5), it consists of two peaks at approx. 14000 and $15500 \mathrm{~cm}^{-1}$. Due to the dichroism of that impurity band in the red, the crystals in question appear dark green or lemon yellow according to the state of polarization of the light used in observing them.

\subsubsection{Absorbing Region}

In the vicinity of the absorption edge (Fig. $7 \mathrm{a}$ and $\mathrm{b}$ ), the absorption spectrum is characterized by a shoulder in the curves of absorption coefficient $\alpha$ at approx. $26000 \mathrm{~cm}^{-1}$ and a strong linear dichroism reaching a peak value $\Delta \alpha=6 \times 10^{3} \mathrm{~cm}^{-1}$ at an energy of $24000 \mathrm{~cm}^{-1}$. In the far ultraviolet up to $6 \mathrm{eV}$, the absorption was too high to be measurable through a crystal thickness of 2.6 micron. From the fact that the dichroic curve goes through zero at approx. $26500 \mathrm{~cm}^{-1}$, it appears that the shoulder at $26000 \mathrm{~cm}^{-1}$ should be composed of two peaks centered on $26500 \mathrm{~cm}^{-1}$, hence with a splitting of the same order of magnitude as that of the impurity band in the red. This splitting of $\sim 1500 \mathrm{~cm}^{-1}$ is observed again between two weak peaks around $22000 \mathrm{~cm}^{-1}$ on the rising part of the dichroic curve. It should therefore be characteristic of the crystal field of $\beta$ - $\mathrm{Eu}_{2} \mathrm{SiO}_{4}$.

\subsubsection{Temperature Dependence of the Absorption Edge}

The colour of the orthorhombic phase of $\mathrm{Eu}_{2} \mathrm{SiO}_{4}$ has been described by Rau [10] as "orange-yellow" and the absorption curve published by Shafer et al. [7] corresponds to an orange colour for thin platelets (Fig. 8). On the other hand, the monoclinic crystals used in this study are lemon yellow in thin platelet form, with the exception of the green ones mentioned above. The yellow colour turns abruptly to orange however at the phase-transition temperature and, on heating slowly, the phase boundary motion normally seen with the help of polarized light (cf. 2.2.) can be directly observed in natural light due to the different colours of those phases. As shown by the inset of Fig. 8 , the colour change is clearly arising from a corresponding discontinuity $(\Delta E=0.11 \mathrm{eV})$ at the $\beta-\alpha^{\prime}$ phase-transition temperature. 
The edge of our $\alpha^{\prime}-\mathrm{Eu}_{2} \mathrm{SiO}_{4}$, however, is in fair agreement with the earlier descriptions of the orthorhombic phase.

The usual temperature shift of the absorption edge has a mean value of $-3 \times 10^{-4} \mathrm{eV} /$ deg between 27 and $135^{\circ} \mathrm{C}$. A comparison of this with the actual temperature dilatation of the lattice and with the change in volume at the phase transition (Table 1) does not allow the assignment of the colour change to a lattice dilatation. Nevertheless, although the Eu-Eu distances do not vary much from one phase to the other, it has been estimated that the rearrangement of the anions around $\mathrm{Eu}_{\mathrm{I}}$, for instance, causes a $30 \%$ change in the volume available to it. From the evidence in the following section that the observed edge should be due to transitions from the localized $4 f$-levels, a widening of the conduction band as a result of the change in coordination and in overall shielding between the cations seems to be responsible for the spontaneous edge shift at the phase-transition temperature.

\subsection{Faraday Rotation}

The Faraday rotation at 300 and $77^{\circ} \mathrm{K}$ for photon energies in the vicinity of the absorption edge has been plotted in Fig. $7 \mathrm{c}$ against the corresponding absorption spectrum. Because of the strong dispersion of the optic axes in that spectral range for the biaxial monoclinic phase, the dispersion of the Faraday effect must in general be measured for directions of propagation away from an optic axis, i.e. in presence of binear birefringence. The dispersion of birefringence was then measured separately on each sample and quantitatively taken into account when computing the true rotations from the measured apparent ones (see [24] for more detail). The general effect of linear dichroism, on the other hand, is to produce a rotation of the plane of vibration of the light towards the azimuth of the leastabsorbed vibration. This rotation is neither a constant for given thickness and wavelength nor a linear function of the magnetic field. However, when the azimuth of the least-absorbed vibration is not in the azimuthal range covered by the rotating vibration, it does not change sign on reversing the magnetic field and can in this manner be eliminated up to second-order effects. This is easily realized in strongly birefringent crystals such as $\beta \cdot \mathrm{Eu}_{2} \mathrm{SiO}_{4}$, since the apparent Faraday rotation is then an oscillating function of thickness where the amplitude, proportional to the applied field, is typically of the order of $1 \mathrm{deg}$.

After applying the above refinements, it appears that the room-temperature curves of the two differently oriented samples considered coincide only in the transparent region of the spectrum. The discrepancies in the absorbing region should therefore arise from an anisotropy of the Faraday effect in the paramagnetic phase of $\beta-\mathrm{Eu}_{2} \mathrm{SiO}_{4}$. Moreover, aithough the low-temperature curve of sample 7 $\left(77^{\circ} \mathrm{K}\right)$ lies higher in magnitude than the room-temperature one, it is by no means inversely proportional to $\left(T-T_{\mathrm{c}}\right)$, which varies approx. by a factor 4 over that temperature range. From the large magnitude of the room-temperature Faraday rotation, one may infer by comparison with the Eu chalcogenides that the optical transitions responsible for the observed edge are from the $4 \hat{j}$-levels to Bloch states with $s$ or $d$ character, i.e. originating from the cations and having rather large amplitudes near the latter. The weak temperature dependence of the Faraday rotation is consistent with the fact that no magnetic red-shift was observed in 


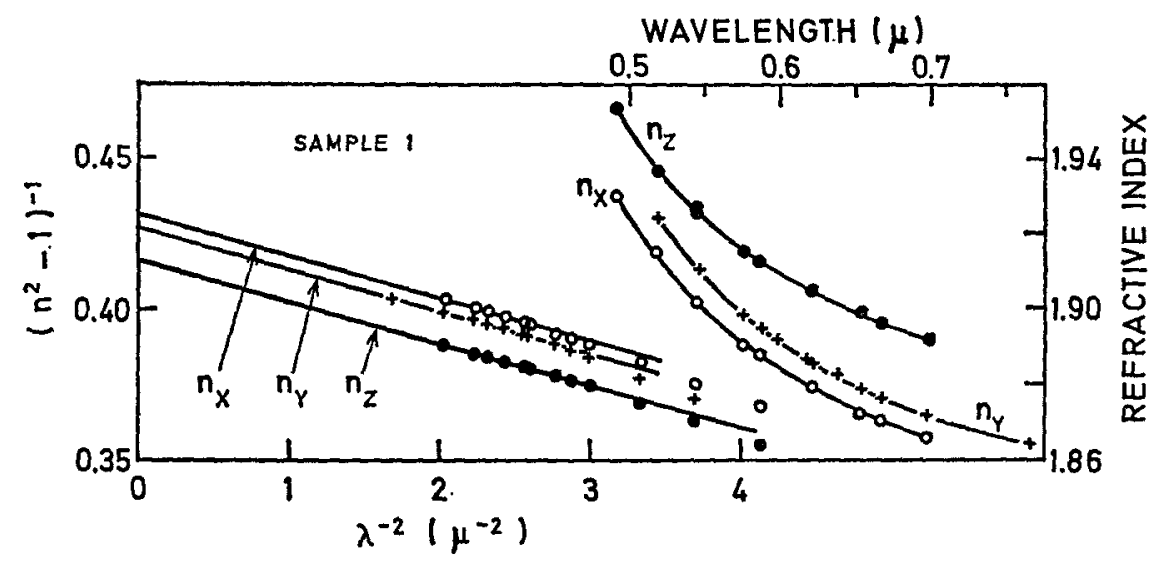

Fig. 9. Principal refractive indices and dispersion relations in $\beta$ - $\mathrm{Eu}_{2} \mathrm{SiO}_{4}$

$\beta-\mathrm{Eu}_{2} \mathrm{SiO}_{4}$ at $\mathrm{He}$ temperature [21]. The reason may be that the spin-orbit splitting of the conduction band becomes accidentally quite small at low temperatures due to the complex character of the interaction.

\subsection{Refractive-Index Ellipsoid}

The petrographic investigation of $\beta-\mathrm{Eu}_{2} \mathrm{SiO}_{4}$ consisted in dispersion measurements of the principal refractive indices, of the optic angle $2 \mathrm{~V}$ and of the position of the optic plane. The refractive index measurements were made in the visible spectrum with the help of interference microscopy on wedge-shaped samples immersed in liquids with refractive indices not too far from those of the crystals, so that the order of the measured path differences could be kept low. The liquids used were mixtures of $\alpha$-monobromonaphthalene, methylene iodide and sulfur $(1.70<n<1.78)$; their dispersion has been separately measured with a ZeissMicroscope-Refractometer.

The values of the principal refractive indices for the sodium line are $n_{\mathrm{x}}=$ $1.886, n_{\mathrm{y}}=1.893$ and $n_{\mathrm{z}}=1.915$. Additional measurements of linear birefringence on various samples, partly with the help of a new method based on light scattering at the sample surface $[25,26]$, showed however that a scatter of up to \pm 5 in the third decimal place of the principal refractive indices can be expected between samples from different production methods. The spectral dependence of the principal refractive indices is shown in Fig. 9, together with their possible fit to a dispersion law of the form

$$
\frac{1}{n^{2}-1}=A+\frac{B}{\lambda^{2}} \text {. }
$$

An analysis of the long-wavelength fit suggests that each refractive index in the red is primarily determined by a single strong absorption line in the far ultraviolet, while the discrepancies in the green appear to be the beginning of a superposed anomalous dispersion pattern arising from the observed absorption edge.

The indicatrix shows the so-called "crossed dispersion", as illustrated in Fig. 10. This type of dispersion allows one to optically recognize monoclinic symmetry 


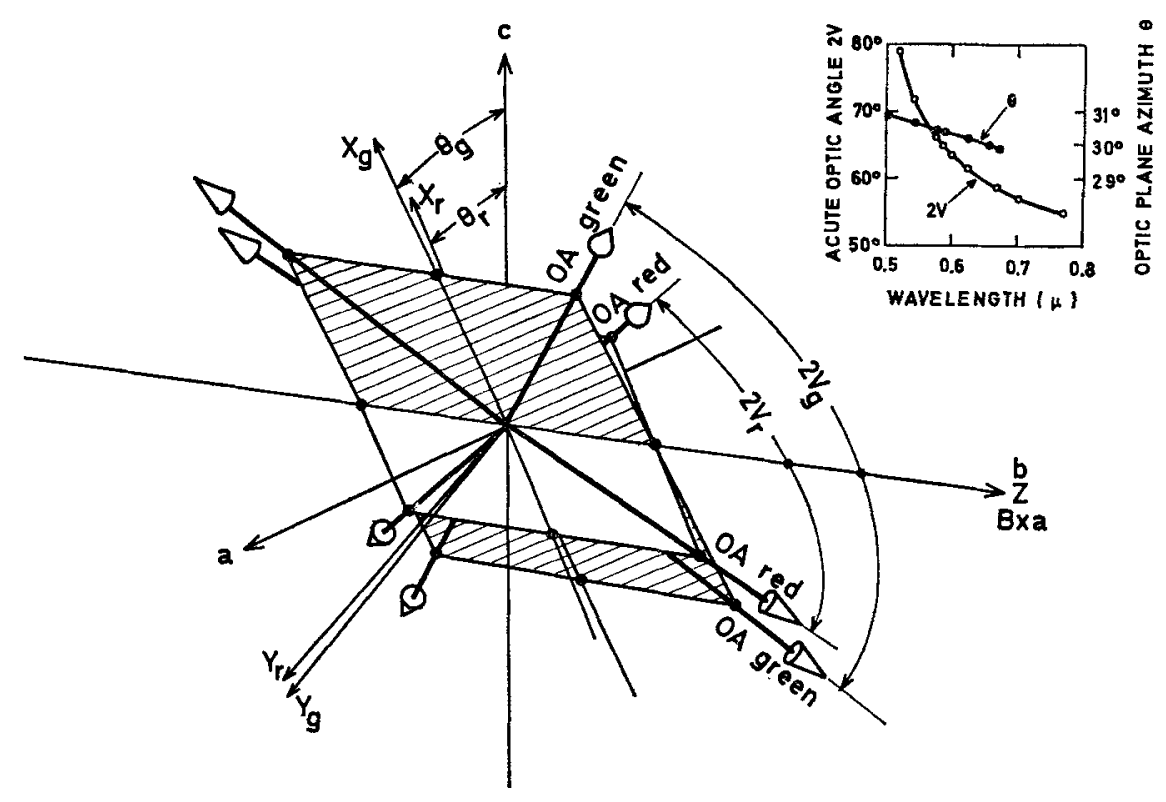

DISPERSION OF THE INDICATRIX IN MONOCLINIC Eu $\mathrm{SiO}_{4}$

Fig. 10. Crossed dispersion of the indicatrix in $\beta$ - $\mathrm{Eu}_{2} \mathrm{SiO}_{4}$. The dispersion of the optic angle and of the ellipsoid orientation are given in the inset

where the $b$ axis lies in the optic plane. It may be noted in particular that each optic axis varies over a range of approx, $15^{\circ}$ in the visible spectrum.

\subsection{Dielectric Constant}

In spite of the fact that the two known phases of $\mathrm{Eu}_{2} \mathrm{SiO}_{4}$ are not ferroelectric due to their crystal symmetry, its static dielectric constant shows some interesting anomalies [27]. The room-temperature value of the order of 25 indicates the presence of strong absorption in the infrared due to lattice vibration and tetrahedron rotations. This is also consistent with the high ionicity mentioned earlier.

A very sharp and unreproducible rise of the dielectric constant over many orders of magnitude has been frequently observed at the ferroelastic-paraelastic phase transition $\left(T_{\mathrm{e}}=165^{\circ} \mathrm{C}\right)$ only on heating, whereas the values in the immediate neighbourhood on either side of $T_{\mathrm{e}}$ show only the small increase typically encountered at structural phase transitions. One must therefore admit the existence of an unstable ferroelectric phase intermediate between the $\beta$ and $\alpha^{\prime}$ phases. It can be tought of as an optical soft mode, possibly from coupled rotations of the tetrahedra in a way which destroys the center of symmetry. The probability for such a mode to be excited may become very high when the tetrahedra collectively switch from their monoclinic to their orthorhombic positions.

Another anomaly of the dielectric constant was also found in the neighbourhood of $10^{\circ} \mathrm{C}$, with peak values (of the order of $10^{2}$ ) dependent on the cooling or heating rate and with half-width of a few degrees. This may be an indication that 
the isomorphism with $\mathrm{Ca}_{2} \mathrm{SiO}_{4}$ is more extended then merely with the $\beta$ and $\mathrm{d}$ phases. It is in fact known $[14,28]$ that the most stable low-temperature phase of $\mathrm{Ca}_{2} \mathrm{SiO}_{4}$ is the $\gamma$ phase, although the $\beta$ phase is easily stabilized by impurities ann the $\beta-\gamma$ phase transition is sluggish, with no definite transition temperature. $\mathrm{I} \alpha$ analogy with the orthorhombic $\gamma$ phase (of the olivine type, $\mathrm{Mg}_{2} \mathrm{SiO}_{4}$ ) of $\mathrm{Ca}_{2} \mathrm{SiO}_{4}$, an incomplete tentative of transition to a similar phase in $\mathrm{Eu}_{2} \mathrm{SiO}_{4}$ may be causing the above anomaly by partially destroying the center of symmetry. The $\beta$ phase is however the only one optically observed so far on our crystals from $165^{\circ} \mathrm{C}$ down to He temperature.

\section{Magnetic Exchange}

From the excellent insulating properties of $\mathrm{Eu}_{2} \mathrm{SiO}_{4}$, the exchange mechanisms which are most likely to occur should be the indirect exchange after Bloembergen and Rowland [31] in the form applied to the Eu chalcogenides by de Graaf and Xavier [32], or the Kramers-Anderson-Goodenough superexchange [33-35]. It may be expected howerer, on account of the high ionicity of $\mathrm{Eu}_{2} \mathrm{SiO}_{4}$, that superexchange, which depends on covalent bonding, could be predominant at the most for long-distance antiferromagnetic interaction, while the Bloembergen-Rowland ferromagnetic interaction should be more important where the Eu-Eu distances are short enough to ensure a significant overlap of the $5 d$ orbitals. An "a priori" estimate of the Bloembergen-Rowland interaction is not possible at this stage because of the lack of information about the energy band structure. Reasoning in terms of the empirical distance dependence of the magnetic interactions (Fig. 11) for the chalcogenide series [36] and considering moreover the results of pressure experiments on $\mathrm{EuO}$ [37-39] and EuS [39], which indicate a stronger distance dependence than in Ref. [36] for a higher ionicity, it is nonetheless possible to utilize the crystallographic information about $\mathrm{Eu}_{2} \mathrm{SiO}_{4}$ in order to explain its magnetic behaviour.

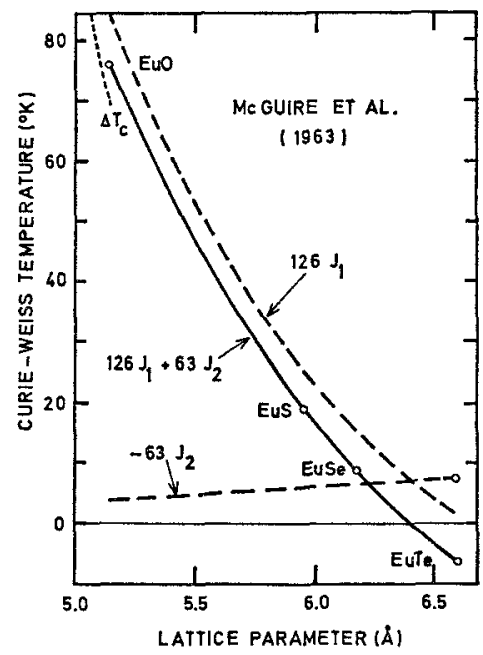

Fig. 11. Empirical distance dependence of the magnetic interactions in the Eu chalcogenide series after Refs. [36-39] 
Table 3. Magnetic data of $\mathrm{Eu}_{2} \mathrm{SiO}_{4}$ crystals

\begin{tabular}{lllllc}
\hline phase & production method & $\theta_{\mathrm{p}}$ & $T_{\mathrm{c}}$ & $\begin{array}{l}\text { saturation } \\
\text { magnetization } \\
{\left[\mu_{\mathrm{B}} / \text { ion }\right)}\end{array}$ & Ref. \\
& & {$\left[{ }^{\circ} \mathrm{K}\right]$} & {$\left[{ }^{\circ} \mathrm{K}\right]$} & 7.5 & 7 \\
\hline$\alpha^{\prime}$ & solid state reaction & $7 \pm 1$ & $\sim 7$ & 6.45 & 30 \\
$\beta$ & I $_{2}$-transport & 5.71 & $5.40 \pm 0.01$ & 6.45 & 30 \\
\hline & HCl-transport & 5.71 & $5.41 \pm 0.01$ & 6.36 & \\
\hline
\end{tabular}

Table 4. Nearest Eu-Eu distances in $\mathrm{Eu}_{2} \mathrm{SiO}_{4}$

\begin{tabular}{|c|c|c|c|c|}
\hline \multirow{3}{*}{$\begin{array}{l}\text { phase } \\
\text { point group symmetry } \\
\text { central cation }\end{array}$} & \multicolumn{2}{|l|}{$\beta$} & \multicolumn{2}{|l|}{$\alpha^{\prime}$} \\
\hline & 1 & & $m$ & \\
\hline & $\mathrm{Eu}_{\mathbf{I}}$ & $\mathrm{Eu}_{\mathrm{II}}$ & $\mathrm{Eu}_{\mathbf{I}}$ & $E u_{I I}$ \\
\hline \multirow{8}{*}{ distances $[\AA]$} & 3.629 & 3.629 & 3.629 & 3.629 \\
\hline & 3.705 & 3.700 & 3.75 & 3.700 \\
\hline & 3.756 & 3.700 & 3.75 & 3.700 \\
\hline & 3.782 & 3.705 & 3.894 & 3.75 \\
\hline & 3.888 & 3.756 & 3.92 & 3.75 \\
\hline & 3.894 & 3.782 & 3.92 & 3.894 \\
\hline & 3.960 & 3.894 & 3.92 & 3.92 \\
\hline & 4.076 & 4.076 & 3.92 & 3.92 \\
\hline
\end{tabular}

Shafer et al. [7] and McGuire et al. [29] have given $7^{\circ} \mathrm{K}$ for the paramagnetic and ferromagnetic Curie temperatures of both powder and monocrystalline samples of the $\alpha^{\prime}$ phase of $\mathrm{Eu}_{2} \mathrm{SiO}_{4}$ stabilized at low temperatures. Landolt [30] looked closely at the magnetic properties of the $\beta$ phase recently and the reliability of his apparatus allowed the results of Table 3 to be obtained. The single crystals used for his measurements were of the same respective batches as samples 2 and 3 in Fig. 6.

Table 4 gives the Eu-Eu distances in $\mathrm{Eu}_{2} \mathrm{SiO}_{4}$. Those of the $\beta$ phase were directly taken from the X-ray data. Each Eu site has the point symmetry 1 in the $\beta$ phase and $\mathrm{m}$ in the $\alpha^{\prime}$ phase. In the latter phase, the distances to atoms lying in the symmetry plane $x=\frac{1}{4}$ were taken unchanged while those to atoms out of that plane, which are mirror images of each other, have been averaged over the corresponding original monoclinic pairs.

The most striking result is that the shortest $\mathrm{Eu}-\mathrm{Eu}$ distance of $3.629 \AA$ is even shorter than in $\mathrm{EuO}(3.64 \AA)$ and that the nearest-neighbour shell (0.4 $\AA$ thick) at a mean distance $d_{n n}=3.81 \AA$ for both Eu sites in both phases is well below the $4.4 \AA$ value suggested by Shafer $e t a l$. [7]. Among the next-nearest neighbours, the surroundings of $\mathrm{Eu}_{\mathrm{I}}$ is rather sharply defined; after an empty gap of $0.8 \AA, 9$ out of $14 n n n$, condensed on a shallow shell $(0.25 \AA$ thick $)$ at approx. $\vec{d}_{n n n}=5.76 \AA$ are seen on the scale model to have a ligand practically in line with the central $\mathrm{Eu}_{\mathrm{I}}$. This is moreover consistent with the above independent estimate of 9 for the coordination. The next-nearest neighbours of EuII however are not so easily visualized and the X-ray data show them to be somewhat more scarcely distributed in the same volume. 
Since the antiferromagnetic exchange is not strongly dependent on distance (Fig. 11), a comparison with the chalcogenide series is probably fair enough. The above value of $\bar{d}_{n n n}$ yields the exchange integral

$$
J_{2} / k=-0.08^{\circ} \mathrm{K} \quad(k=\text { Boltzmann's constant }) .
$$

For determining the ferromagnetic exchange integral in $\mathrm{Eu}_{2} \mathrm{SiO}_{4}$ however, the empirical curve of McGuire et al. [36] is probably of little use since the ionicity of the chalcogenides is lower than that of the silicate and moreover steadily decreases from $\mathrm{EuO}$ to EuTe. From the fact that $\mathrm{Eu}_{2} \mathrm{SiO}_{4}$ has a higher ionicity than $\mathrm{EuO}$ and that the degree of ionicity appears to determine the steepness of the ferromagnetic-exchange curves in Fig. 11, one may assume a stronger distance dependence than the one given from the pressure experiments on $\mathrm{EuO}$ (dotted curve). One is then led to the conclusion that only a small fraction of the irregularly positioned nearest neighbours should contribute significantly to the ferromagnetic exchange. In determining this fraction, one notices that the $\mathrm{m}$ symmetry of the Eu sites in the $\alpha^{\prime}$ phase limits the first choice between 1 neighbour or 3 neighbours, since the second and the third ones should bring exactly the same contribution. The fact that the Curie temperature and the first values of $d_{n n}$ are practically the same for both phases means that the same number of contributing neighbours have to be considered in both phases, where the first one should have a value of $J_{1}$ comparable to the $J_{1}$ value in EuO. By assuming three contributing nearest neighbours with a mean value $J_{1} / k=0.45^{\circ} \mathrm{K}$, the molecular field approximation gives

$$
\theta_{\mathrm{p}}=\frac{2}{3 k} S(S+1)\left(3 J_{1}+9 J_{2}\right)=6^{\circ} \mathrm{K},
$$

in fair agreement with the data of 'Table 3.

It appears therefore that in spite of nearest-neighbour distances much closer to those of $\mathrm{EuO}$ than those of EuSe, the low Curie point of $\mathrm{Eu}_{2} \mathrm{SiO}_{4}$ is the result of the irregular nearest-neighbour shell, which produces a strong discrimination between the nearest neighbours contributing significantly to the ferromagnetic exchange. The smaller number (3) of the latter than in $\mathrm{EuO}(12)$, the larger value of $J_{2}$ and also a larger number $(\sim 9)$ of antiferromagnetically coupled nextnearest neighbours than in $\mathrm{EuO}(6)$ all contribute to lower the Curie temperature of $\mathrm{Eu}_{2} \mathrm{SiO}_{4}$ as compared to $\mathrm{EuO}$.

\section{Conclusion}

The knowledge of the crystal structure of $\mathrm{Eu}_{2} \mathrm{SiO}_{4}$ has allowed a first explanation of its magnetic behaviour to be given. It turns out that this substance is a striking example of the drastic influence which the structure can have on the exchange properties.

To our knowledge, $\mathrm{Eu}_{2} \mathrm{SiO}_{4}$ is the first substance to show simultaneously ferromagnetism and ferroelasticity. It may be of interest to study the coupling between both phenomena. From the large difference between the ferromagnetic Curie temperature $T_{\mathrm{c}}$ and the ferroelastic one $\left(T_{\mathrm{e}}\right)$, it is expected that the influence of ferromagnetic ordering on the ferroelastic domain structure should arise from magnetostriction through the magnetoelastic constants. There may however 
exist materials where $T_{\mathrm{c}}$ in the ferroelastic phase is larger than $T_{\mathrm{c}}$ in the paraelastic one. In such cases, the spontaneously appearing ferroelastic phase at $T_{\mathrm{e}}$ $\left(T_{\mathrm{c} \text {,parael }}<T_{\mathrm{e}}<T_{\mathrm{c} \text {, ferroel }}\right)$ would induce magnetic ordering through a change in interatomic distances, and hence in the exchange integrals $J_{1}$ and $J_{2}$. This might even be the case for $\mathrm{Eu}_{2} \mathrm{SiO}_{4}$ if a $\gamma$ phase isomorphous with $\gamma-\mathrm{Ca}_{2} \mathrm{SiO}_{4}$ exists at low temperatures, as suggested by the dielectric constant anomaly at $10^{\circ} \mathrm{C}$. Since the $\beta-\gamma$ phase transition involves a $12 \%$ increase in volume, the $\gamma$ phase of $\mathrm{Eu}_{2} \mathrm{SiO}_{4}$ should be antiferromagnetic or paramagnetic at low temperatures. It may be also of interest to study the ferroelastic-paraelastic phase transition at $165^{\circ} \mathrm{C}$ in Raman scattering in order to identify the soft mode associated with that transition.

The isomorphism between $\mathrm{Eu}_{2} \mathrm{SiO}_{4}$ and $\mathrm{Ca}_{2} \mathrm{SiO}_{4}$ should make it possible to realize complete miscibility of both substances in a given phase. In dilute solutions of $\mathrm{Eu}_{2} \mathrm{SiO}_{4}$ in $\mathrm{Ca}_{2} \mathrm{SiO}_{4}$, it may be possible to identify the optical transitions responsible for the observed absorption edge through an eventual resolving of the 4 multiplet in absorption.

Acknowledgements. I wish to thank Prof. G. Busch sincerely for the encouraging interest taken in this investigation and for stimulating suggestions. Special thanks go also to Prof. F. Laves, Prof. W. Baltensperger and Prof. A. A. Samokhvalov for instructive discussions. I feel much indebted to Dr. E. Kaldis for supplying the crystals, to Dr. J. Felsche for his generous contribution with the X-ray data and to Mr. M. Landolt for his magnetic measurements and for beneficial discussions. The prompt assistance of Mr. A. Heusser and Mr. W. Schmid in many apparative problems was greatly appreciated. This work was supported by the Swiss National Fund for Scientific Research.

\section{References}

1. Tsubokawa, I.: J. Phys. Soc. Japan 15, 1664 (1960).

2. Matthias, B. T., Bozorth, R. M., Van Vleck, J. H.: Phys. Rev. Letters 7, 160 (1961).

3. Busch, G., Junod, P., Risi, M., Vogt, O.: Proc. Int. Conf. Semi-Conductors, Exeter, 727 (1962).

4. Busch, G., Junod, P., Wachter, P.: Phys. Letters 12, 11 (1964).

5. Garton, G., Hukin, D. A.: paper given at the 3rd Rare Earth Conf., Florida, Apr. 21-24, 1963; Rare Earth Research II (Vorres), Gordon \& Breach, N. Y. 1964, p. 3.

6. Rau, R. C.: ibid., p. 117.

7. Shafer, M. W., MoGuire, T. R., Suits, J. C.: Phys. Rev. Letters 11, 251 (1963).

8. Kaldis, E., Verreault, R.: J. Less-Common Metals 20, 177 (1970).

9. Busch, G., Kaldis, E., Verreault, R., Felsche, J.: Materials Res. Bull. 5, 9 (1970).

10. Rau, R. C.: Acta cryst. 17, 1483 (1964).

11. Midgley, C. M. : Acta cryst. 5, 307 (1952).

12. Yamaguchi, G., Miyabe, H., Amano, K., Komatsu, S.: J. Ceram. Soc. Japan 65, 99 (1957).

13. Felsche, J.: private communication.

14. Smith, D. K., Majumdar, A., Ordway, F.: Acta cryst. 18, 787 (1965).

15. Busch, G., Landolt, M., Verreault, R.: to be published.

16. Aizu, K.: J. Phys. Soc. Japan 27, 387 (1969).

17. Gerth, G., Kienle, P., Luchner, K.: Phys. Letters 27A, 557 (1968).

18. Gerth, G.: masters thesis, unpublished.

19. Pink, H.: private communication.

20. Groll, G.: private communication.

21. Busch, G., Streit, P., Wachter, P.: Helv. phys. Acta 42, 930 (1969).

22. Dieke, G. H., Crosswhite, H. M. : Appl. Optics 2, 675 (1963).

23. Wachter, P.: private communication.

24. Becquerel, J.: Z. Phys. 52, 342 (1929). 
25. Busch, G., Verreault, R.: to be published in Acta cryst.

26. Verreault, R.: to be published in Z. Krist.

27. Kaldis, E.: private communication.

28. O'Daniel, H., Tscheischwili, L.: Z. Krist. 104, 124 (1942).

29. McGuire, T. R., Shafer, M. W.: J. appl. Phys. 35, 984 (1964).

30. Landolt, M.: private communication.

31. Bloembergen, N., Rowland, T. J.: Phys. Rev. 97, 1679 (1955).

32. de Graaf, A. M., Xavier, R. M. : Phys. Letters 18, 225 (1965).

33. Kramers, H. A.: Physica 1, 182 (1934).

34. Anderson, P. W.: Phys. Rev. 79, 350 (1950); 115, 2 (1959).

35. Goodenough, J. B.: Phys. Rev. 100, 594 (1955); Phys. Chem. Solids 6, 287 (1958).

36. MeGuire, T. R., Argyle, B. E., Shafer, M. W., Smart, J. S.: J. appl. Phys. 34, 1345 (1963).

37. Stevenson, R., Robinson, M. C.: Can. J. Phys. 43, 1744 (1965).

38. McWhan, D. B., Sauers, P. C., Jura, G.: Phys. Rev. 143, A 385 (1966).

39. Schwob, P.: Phys. kondens. Materie 10, 186 (1969).

\section{R. Verreault}

Dept. of Physics

Univ. du Quebec à Chicoutimi

930 Est, Rue Jacques-Cartier

Chicoutimi, Quebec, Canada 